Boletín de la Sociedad Geológica Mexicana

VOLUMEN 62, NÚM. 3, 2010, P. 345-357

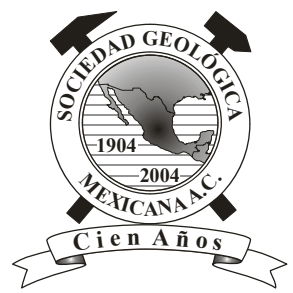

\title{
Evolución Paleolimnológica del Lago Cuitzeo, Michoacán durante el Pleistoceno-Holoceno
}

\author{
Isabel Israde Alcántara ${ }^{1, *}$, Rodrigo Velázquez-Durán ${ }^{3}$, Ma. Socorro Lozano García ${ }^{2}$, James \\ Bischoff $^{4}$, Gabriela Domínguez Vázquez ${ }^{5}$, Víctor Hugo Garduño Monroy ${ }^{1}$ \\ ${ }^{1}$ Departamento de Geología, Instituto de Investigaciones Metalúrgicas, Universidad Michoacana de San Nicólas Hidalgo. Edif. U, \\ Ciudad Universitaria, C. P. 58060, Morelia, Michoacán \\ ${ }^{2}$ Departamento de Paleontología, Instituto de Geología, Universidad Nacional Autónoma de México. Ciudad Universitaria, C.P. \\ 04510, México, Distrito Federal. \\ ${ }^{3}$ Centro de Investigaciones en Ecosistemas, Universidad Nacional Autónoma de México, Campus Morelia. Antigua Carretera a \\ Pátzcuaro No. 8701, Col. Ex-Hacienda de San José de La Huerta C. P. 58190, Morelia Michoacán. \\ ${ }^{4}$ United States Geological Survey, 345 Middlefield Road, Menlo Park, 94025-3561, California. \\ ${ }^{5}$ Facultad de Biología, Universidad Michoacana de San Nicólas Hidalgo. Edificio R. Ciudad Universitaria, C. P. 58060 , Morelia, \\ Michoacán. \\ *isaisrade@gmail.com
}

\begin{abstract}
Resumen
El lago de Cuitzeo, situado en la región volcánica de la Meseta Tarasca al norte de Michoacán, se formó dentro de una cuenca tectónica y es el segundo vaso lacustre más grande de México. Este estudio se basa en un núcleo de $27 \mathrm{~m}$ perforado en el depocentro del lago y en cuatro trincheras de las zonas proximales recientemente expuestas por el retroceso del lago. Las arcillas, limos y lodos diatomáceos fueron el material dominante en el núcleo, mientras que en las trincheras dominan facies fluviales intercaladas con depósitos volcanoclásticos retrabajados, depósitos de cenizas, surges y tobas de caída aérea depositados en agua y en paleosuelos. El control cronológico con fechas de radiocarbono se obtuvo hasta los 11 metros de profundidad (42 ka a. P.) infiriéndose que la base del núcleo tiene aproximadamente $120 \mathrm{ka}$ a. P. Los resultados de los análisis de diatomeas, polen, mineralogía, geoquímica del núcleo de $27 \mathrm{~m}$ y macropartículas carbonizadas de las trincheras muestran que el lago experimentó distintos cambios ambientales. La química de los sedimentos y los análisis de las fluctuaciones de diatomeas sugieren que antes de los $50 \mathrm{ka}$ a. P. el lago mantuvo condiciones de aguas bajas y diluidas. Un ascenso del nivel lacustre o condiciones más diluidas, ocasionados por un flujo de agua dulce, se observan en cinco periodos (ca. 100 ka a. P., ca. 50 ka a.P., de 44 ka a 42 ka a. P., de 32 ka a 31 a. P. y de 20 ka a 14 ka a. P.) con Stephanodiscus spp., Aulacoseira spp. y especies del grupo Fragilaria como taxa dominantes, lo cual fue corroborado por los altos valores de Cyperaceae y Typha. A partir de $45 \mathrm{ka}$ a. P. se registran episodios de aridez en el lago con una tendencia a la disminución de profundidad, convirtiéndose en un lago de aguas bajas, alcalino y de alta concentración iónica. Picos de incremento de la concentración iónica son evidentes de $45 \mathrm{ka} \mathrm{a} 43 \mathrm{ka}$ a. P., de $38 \mathrm{ka}$ a $35 \mathrm{ka}$ a. P, de $31 \mathrm{ka}$ a $25 \mathrm{ka}$ a. P. y de $12 \mathrm{ka} \mathrm{a} 2 \mathrm{ka}$ a. P. Depósitos de tefra se observan a los ca. 80 ka a. P, ca. 50 ka a. P., ca. 35 ka a. P y ca. 25 ka a. P. Durante el Holoceno medio ( $c a .5$ ka a. P.), y por un corto periodo, se presenta flora de ambientes más diluidos. Sin embargo, de 2 ka a. P. al presente la flora de diatomeas se encuentra dominada por Discostella meneghiniana, lo que indica una clara tendencia a la disminución del nivel de las aguas del lago, con un incremento de la turbidez y la concentración iónica. Los indicadores de las partículas carbonizadas señalan una fuerte relación entre la ocurrencia de incendios y la actividad volcánica, la cual disminuye durante el Holoceno tardío. Se observa una interrupción en la sedimentación entre ca. 8 ka y ca. $12 \mathrm{ka}$ a. P. Este hiato también está representado en un núcleo extraído en la zona litoral del norte del lago de Zirahuén.
\end{abstract}

Palabras clave: Lago de Cuitzeo, México, diatomeas, polen, niveles lacustres, Cuaternario. 


\begin{abstract}
Lake Cuitzeo, located in a volcanic region in northern Michoacan called the Tarascan Plateau, was formed in a tectonic basin and is the second largest lake in Mexico. This study is based on a $27 \mathrm{~m}$ long core, drilled in the depocenter of the lake, and four trenches in the proximal areas recently exposed by the retreat of the lake. The predominant materials in the core are clays, silts and diatomaceous muds, while in the trenches fluvial facies are interlayered with deposits of reworked volcaniclastics, ash, pyroclastic surges and air-fall tuffs that were deposited in water or in paleosoils. Chronological control is based on radiocarbon dates for the first part of the core down to $11 \mathrm{~m} \mathrm{(42} \mathrm{ky} \mathrm{BP),} \mathrm{and} \mathrm{an} \mathrm{age} \mathrm{of} \mathrm{ca.} 120 \mathrm{ky} \mathrm{BP}$ is inferred for the base of the core. Results from diatom, pollen, mineralogy, geochemistry and macro-charcoal particle analyses from the trenches show that Lake Cuitzeo experienced several environmental changes. Sediment chemistry and diatom fuctuations suggest that before $50 \mathrm{ky}$ BP it was a shallow freshwater lake. Five periods of increasing lake level or more diluted conditions were caused by freshwater entry to the lake (ca. $100 \mathrm{ky} \mathrm{BP,} \mathrm{ca.} 50 \mathrm{ky} B P$, from $44 \mathrm{ky}$

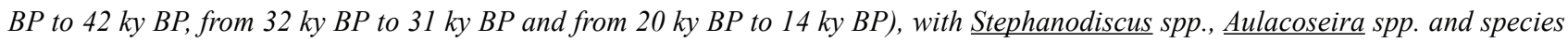
from the Fragilaria group as dominant taxa, which coincided with high values of Cyperaceae and Typha. From $45 \mathrm{ky} B P$ the lake has

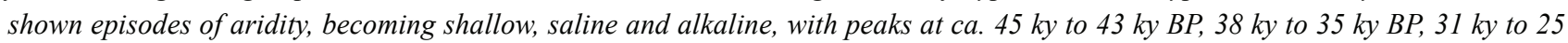
$\mathrm{ky} B P$, and $12 \mathrm{ky}$ to $2 \mathrm{ky}$ BP. Tephra deposits are observed at ca. $80 \mathrm{ky}$ BP, ca. $50 \mathrm{ky} \mathrm{BP,ca.} 35 \mathrm{ky} \mathrm{BP}$ and ca. $25 \mathrm{ky}$ BP. During the middle Holocene ( $c a .5 \mathrm{ky} B P$ ), for a short period of time the flora indicates more diluted environments, however from $2 \mathrm{ky} B P$ to the present diatom flora is dominated by Discostella meneghiniana, which is indicative of shallow, turbid and saline water. Macro-charcoal particle analysis indicates a strong relation between fire events and the volcanic activity in the area, which decreased during the late Holocene. An interruption in the sedimentation is observed between ca. $8 \mathrm{ky}$ and ca. $12 \mathrm{ky}$ BP. This hiatus also is represented in a core from the northern shore of Zirahuén Lake.
\end{abstract}

Keywords: Cuitzeo Lake, Mexico, diatoms, pollen, lake levels, Quaternary.

\section{Introducción}

Los lagos conforman potenciales almacenes de materiales alogénicos y autigénicos, por lo que poseen un registro continuo de los eventos volcánicos, tectónicos y climáticos que han ocurrido a nivel local y regional. Las depresiones lacustres más conocidas por su extensión son las depresiones de Ixtlahuaca, Acámbaro, Acambay, Sayula y Charo, ahora ya secas, pero que en sus depósitos testimonian que albergaron una abundante flora y fauna (IsradeAlcántara et al., 2010). Los lagos que aún permanecen activos son los de Pátzcuaro, Zacapu, Cuitzeo y Chapala, los cuales tienen como común denominador que en sus márgenes (generalmente el sur) afloran sedimentos lacustres levantados por efecto de la tectónica y/o el vulcanismo (Israde-Alcántara y Garduño-Monroy, 1999).

Los sistemas lacustres más antiguos del centro de México son Chapala y Cuitzeo, que se originaron en algún momento de quiescencia del vulcanismo durante el Mioceno superior (Garduño-Monroy y Negrín, 1992). Sin embargo, los lagos activos de Michoacán que han sido ampliamente estudiados con fines paleolimnológicos cubriendo rangos de tiempo desde el Pleistoceno superior y durante el Holoceno son: Pátzcuaro, Zirahuén, Zacapu y Tacámbaro (Watts y Bradbury, 1982; Bradbury, 2000; Ortega et al., 2002, 2010; Davies et al., 2005; Metcalfe et al., 2007; Israde-Alcántara et al., 2009). En particular, el lago de Cuitzeo tiene un basamento conformado por lavas de composición andesítica que fueron emitidas hace $8 \pm 0.2 \mathrm{Ma}$ (CFE, 1986). La actividad tectónica produjo la fragmentación de las lavas emitidas, generando un graben rellenado por depósitos volcánicos y sedimentarios, según los transectos gravimétricos realizados a todo lo largo y ancho del lago (Arredondo, 1983).

Desde el punto de vista paleolimnológico, Cuitzeo ha sido el único lago estudiado a detalle desde el Mioceno hasta el Reciente (Israde-Alcántara y Garduño-Monroy, 1999; Israde-Alcántara et al., 2002). Sus depósitos sedimentarios se componen de materiales biogénicos (diatomeas y polen), líticos (arcillas, limos arenosos y diatomitas que se intercalan con depósitos de cenizas volcánicas) y, con menos abundancia, depósitos de precipitación química (yesos). Los sedimentos en conjunto permitieron reconstruir $c a .120 \mathrm{ka}$ de su historia, basados en resultados del análisis de polen, geoquímica, diatomeas y partículas carbonizadas.

\section{Materiales y métodos}

El lago de Cuitzeo (Figura 1) se encuentra ubicado en las coordenadas $19^{\circ} 53^{\prime} 15^{\prime \prime} \mathrm{N}-20^{\circ} 04^{\prime} 34^{\prime \prime} \mathrm{N}$ y $100^{\circ} 50^{\prime} 20^{\prime \prime}$ O - 101'19'34" O) a $1880 \mathrm{msnm}$, forma parte de una serie de depresiones tectónicas alineadas en dirección E-O en las que se han establecido sistemas lacustres, algunos de ellos ya desaparecidos por efecto del vulcanismo y la tectónica que han contribuido a su colmatación.

En el lago de Cuitzeo se extrajo un núcleo de $27.4 \mathrm{~m}$ de longitud, utilizando un muestreador long gear a presión neumática, con el que se recuperaron tramos de $100 \mathrm{~cm}$ de largo, los cuales fueron cubiertos con cera y manta de 
cielo para evitar su desecación. Se tomaron muestras de los núcleos en intervalos de $c a .10 \mathrm{~cm}$ para los diferentes análisis (polen, diatomeas, geoquímica, carbono orgánico, isótopos y susceptibilidad magnética). Se excavaron cuatro trincheras hacia el norte del lago para determinar en estas facies distales la ocurrencias de incendios locales (Figura 1).

\subsection{Fechamientos}

Para contar con un marco cronológico se fecharon 18 niveles orgánicos por el método de ${ }^{14} \mathrm{C}$ y espectrometría de masas con acelerador (AMS) realizados en la Universidad de Arizona y en el laboratorio Lawrence Livermore, California (Figura 2, Tabla 1). Se utilizaron las fechas de radiocarbono sin calibrar debido a la amplitud de la variación en las tasas de sedimentación. La tasa de sedimentación más baja fue de $0.0016 \mathrm{~mm} / \mathrm{año}$, se presenta entre 8830 y 14720 años a.P. y corresponde a 5890 años de depósito en $10 \mathrm{~cm}$, indicando una fase de baja tasa de sedimentación o bien de intensa erosión de la cuenca y del lago de Cuitzeo.

\subsection{Polen}

Las muestras para el análisis polínico fueron trituradas en un mortero metálico, y sometidas a dos lavados con Sigmaclin. Posteriormente, se le agregó una pastilla con esporas marcadoras de Lycopodium clavatum con media de 12543 esporas por tableta. Sucesivamente se adicionaron $10 \mathrm{~mL}$ de ácido clorhídrico al 10\%, eliminando con esto la mayor cantidad de carbonatos. Cuando las muestras alcanzaron la neutralidad, se les agregó ácido fluorhídrico por 24 horas; para eliminar la sílice de las muestras. Una vez neutralizada la muestra se acetolizó durante 15 minutos a baño María. La muestra acetolizada se tiñó con una gota de safranina y finalmente el montaje se realizó en gelatina glicerinada (Erdtman, 1969). Se llevó un registro fotográfico y se identificó el material polínico fósil mediante el uso de la bibliografía especializada.

\subsection{Diatomeas}

Para el análisis de diatomeas se recuperaron de $0.5 \mathrm{~g}$ de sedimento seco que se procesaron con $\mathrm{HCl}$ al $10 \%$ y, una vez enjuagado, se agregó $\mathrm{H}_{2} \mathrm{O}_{2}$ para realizar la digestión de la materia orgánica a temperaturas inferiores a $100{ }^{\circ} \mathrm{C}$. Una vez neutralizada la muestra se tomó una alícuota de 3 $\mu \mathrm{L}$, que se dejó secar sobre un cubreobjetos a temperatura ambiente. Para el montaje se utilizó Nafrax con alto índice de refracción $(I R=1.73)$. Se contaron 500 valvas por muestra. Los taxa fueron inventariados y fotografiados. La taxonomía de las diatomeas y la información de las condiciones ecológicas se basó en Hustedt (1930), Round

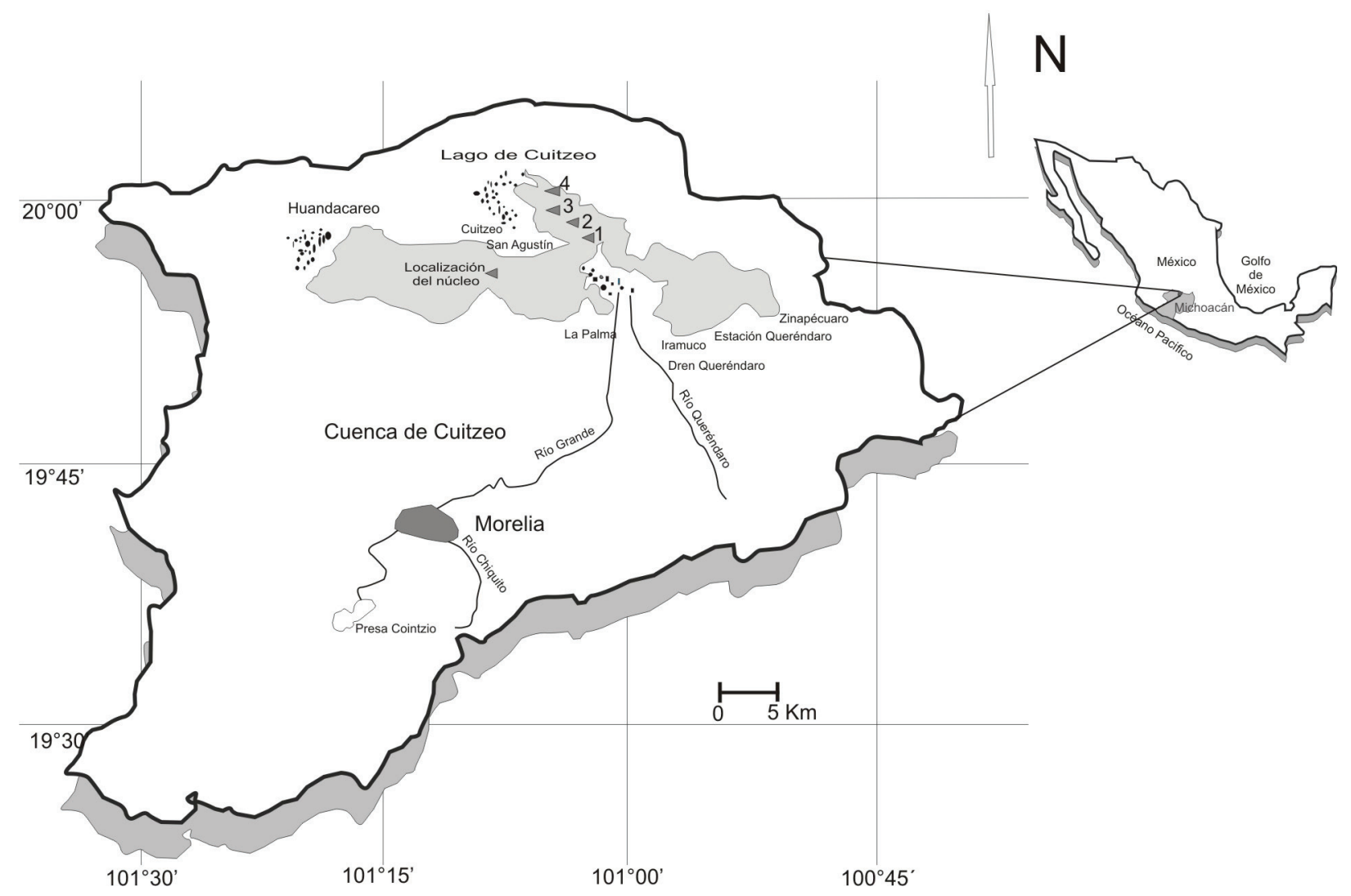

Figura 1. Localización del lago de Cuitzeo, del núcleo extraído y de las trincheras excavadas. 
(1981), Gasse (1986), Bradbury (2000), Israde-Alcántara (1995), Hartley et al. (1996), Krammer y Lange-Bertalot (1986, 1988, 1991a, 1991b), Rumrich et al. (2000), y LangeBertalot (2001).

\subsection{Micropartículas carbonizadas}

Se utilizó la técnica de Laird y Campbell (2000), la cual consiste en tomar $1 \mathrm{~cm} 3$ de sedimentos y dejarlos en $\mathrm{KOH}$ al $10 \%$ por 24 horas. Después se desechó el sobrenadante y se adicionó peróxido de hidrógeno al $30 \%$ por 3 horas. Las muestras se tamizaron utilizando un filtro de $80 \mu \mathrm{m}$. El material retenido en la malla se colocó en cajas de Petri para proceder a su conteo.

\subsection{Análisis geoquímicos}

Los análisis geoquímicos para contenido de $\mathrm{Al}, \mathrm{Mg}$, $\mathrm{Na}$ y K, se realizaron en el laboratorio SGS-CANADA Inc. Por su parte, las muestras para carbono inorgánico total (CIT) y carbono orgánico total (COT) se procesaron en el Servicio Geológico de Estados Unidos (Menlo Park, California); utilizando un coulómetro UIC-CM5014 que funciona con el método de titulación. La muestra se calcinó a $900{ }^{\circ} \mathrm{C}$ determinando el porcentaje de carbono total, para lo cual se pesaron $0.022 \mathrm{~g}$ de sedimentos secos y molidos. Posteriormente, en el módulo de acidificación UIC CM5130, se cuantificó el carbono inorgánico total (CIT).

Los análisis de difracción de rayos $\mathrm{X}$ se realizaron en el Instituto de Investigaciones Metalúrgicas de la Universidad
Michoacana de San Nicolás de Hidalgo, en un difractómetro Siemens 5000 de radiación monocromática de $\mathrm{Cu} \mathrm{K} \alpha(\lambda=$ $1.5406 \AA$ ) a $30 \mathrm{kV}$ y $25 \mathrm{~mA}$, con una constante $\mathrm{t}=0.61 .0$ $\mathrm{s}$ y una constante de paso angular de $0.02^{\circ}$ para $2 \theta$.

\section{Resultados y discusión}

Se realizaron 18 fechamientos en los primeros $9 \mathrm{~m}$ de profundidad, obteniéndose una edad de 42400 años (Figura 2, Tabla 1) la cual representa el límite para el fechado por radiocarbono; no ha sido posible obtener cenizas que contengan material susceptible para fechar hacia la base del núcleo. Si se considera una tasa de sedimentación homogénea a partir de los $9 \mathrm{~m}$, la edad de la base del núcleo alcanzaría los ca. $120 \mathrm{ka}$ a.P. alcanzando el término del último interglacial (Figura 2).

Se registraron 31 taxa de diatomeas con porcentajes mayores al 5\% y 187 taxa polínicos a lo largo de la secuencia (Figura 4). El registro de diatomeas muestra que Staurosira spp. es el taxón más importante desde la base hasta $c a$. $17.6 \mathrm{ka}$ a.P., mientras que desde $8 \mathrm{ka}$ y hasta el presente Discostella meneghiniana se vuelve el taxón dominante. Durante el Cuaternario tardío se presentan cinco fases relativamente húmedas ( $c a .100 \mathrm{ka}, \mathrm{ca} .50 \mathrm{ka}$, de $44 \mathrm{ka} \mathrm{a} 42$ ka a.P., de 31 ka a 32 ka y de 20 ka a 14 ka a.P.), inferidas por la presencia de Stephanodiscus spp., Aulacoseira spp. y especies del grupo Fragilaria, diatomeas que en conjunto indican condiciones diluidas y ambientes con tendencia a ser más profundos.

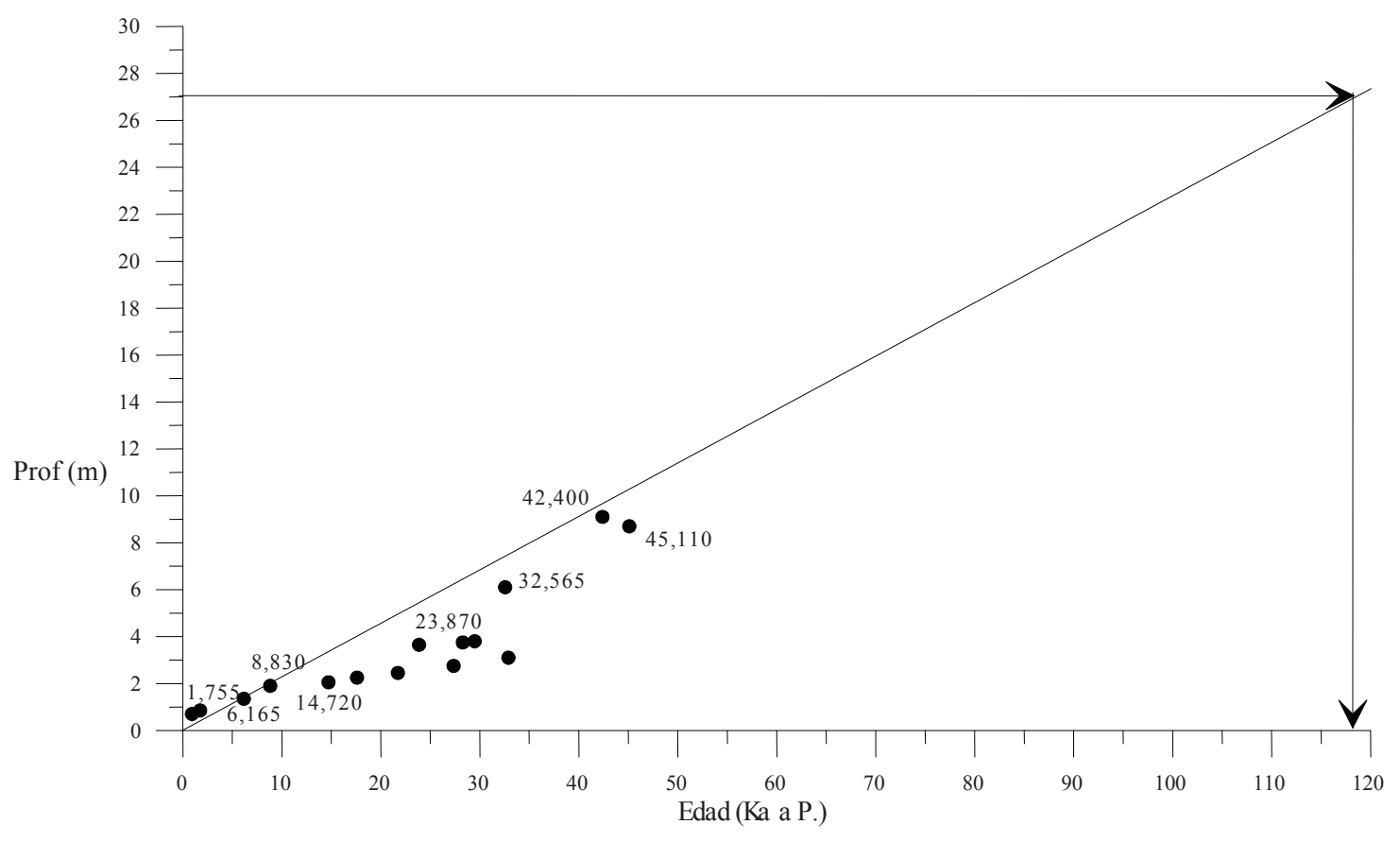

Figura 2. Curva de edad del núcleo largo de Cuitzeo. 
Tabla 1. Fechamientos del núcleo del depocentro del lago de Cuitzeo y edades calibradas con el programa CALIB REV6.0 (Stuiver y Reimer, 1993).

\begin{tabular}{cccccl}
\hline $\begin{array}{c}\text { Profundidad } \\
(\mathrm{m})\end{array}$ & $\begin{array}{c}\text { Fechas } \\
\text { radiocarbono }\end{array}$ & $+/-$ & Sigma & Probabilidad & \multicolumn{1}{c}{ Edades calibradas } \\
\hline 0.7 & 930 & 55 & 2 sigma & 1.000 & cal AD 1016- 1218 \\
0.85 & 1755 & 115 & 2 sigma & 1.000 & cal AD 25- 543 \\
1.35 & 6165 & 70 & 2 sigma & 1.000 & cal BC 5300- 4943 \\
1.9 & 8830 & 215 & 2 sigma & 0.994 & cal BC 8483- 7512 \\
2.05 & 14720 & 50 & 2 sigma & 0.926 & cal BC 16119- 15678 \\
2.25 & 17605 & 215 & 2 sigma & 1.000 & cal BC 19527- 18422 \\
2.45 & 21730 & 70 & 2 sigma & 0.965 & cal BC 24397- 23788 \\
2.75 & 27360 & 130 & 2 sigma & 0.965 & cal BC 29811-29286 \\
3.1 & 32940 & 190 & 2 sigma & 1.000 & cal BC 36511- 34900 \\
3.35 & 15500 & 130 & 2 sigma & 1.000 & cal BC 16981- 16569 \\
3.65 & 23870 & 100 & 2 sigma & 1.000 & cal BC 27248- 26348 \\
3.75 & 28289 & 120 & 2 sigma & 1.000 & cal BC 31160- 30002 \\
3.8 & 29490 & 190 & 2 sigma & 1.000 & cal BC 32718- 31572 \\
6.1 & 32565 & 2855 & 2 sigma & 1.000 & cal BC 40356- 29541 \\
8.7 & 45110 & 940 & 2 sigma & 1.000 & cal BC 48051- 44522 \\
9.1 & 42400 & 1000 & 2 sigma & 1.000 & cal BC 45458- 41955 \\
\hline
\end{tabular}

Los taxa polínicos encontrados (Figuras 5 y 6 ) fueron agrupados según sus afinidades ecológicas. Abies es un taxón representativo del bosque de Abies. En los bosques de pino-encino se incluyó a Pinus, CupressusJuniperus, Quercus, Alnus y Buddleia, mientras que los bosques mesófilos fueron caracterizados por taxa como Liquidambar, Juglans, Fraxinus, Corylus-Carpinus, Betula. Los taxa arbustivos y herbáceos que indican la presencia de condiciones perturbadas y abiertas son pastos y compuestas, incluyendo Ambrosia, mientras que las Chenopodiaceae-Amaranthaceae son elementos comunes de áreas de disturbio así como de condiciones pantanosas. Los taxa asociados a condiciones lacustres son Cyperus y Typha. En el caso de las algas, las más comunes fueron Pediastrum y Botryococcus.

En los párrafos siguientes se discuten las fluctuaciones de las comunidades vegetales (diatomeas y polen) para cada zona del núcleo y se asocian con el registro de COT y con la mineralogía, que en conjunto sugieren los paleoambientes y variaciones climáticas del Pleistoceno tardío-Holoceno del lago de Cuitzeo (Figuras 3a, 3b y 4, Tabla 2). Los datos geoquímicos indican que la mayor parte de los componentes terrígenos de los sedimentos son de composición andesítica y dentro de los componentes autogénicos los valores elevados de $\mathrm{Mg} / \mathrm{Al}$ son más abundantes en el intervalo Holocénico del núcleo, mientras que altos valores de la relación $(\mathrm{K}+\mathrm{Na}) / \mathrm{Al}$ corresponden a depósitos de tefras (ca.80 ka, ca.50 ka y ca.35 a 25 ka a.P.; Figura 4).

\subsection{Análisis de paleoambientes}

\subsubsection{Zona I (27.30 m. a $26.30 \mathrm{~m}$; ca. $130 \mathrm{ka} \mathrm{a} 90 \mathrm{ka}$ a.P.)}

Esta zona comprende el periodo entre $c a .130$ ka y 90 ka a.P, el cual se estima que corresponde al final del último interglacial. De acuerdo con los conjuntos polínicos se deduce la presencia de un bosque templado del tipo pinoencino (Figura 5), mientras que en los lugares abiertos
Tabla 2. Frecuencia de aparición de minerales selectos determinados por DRX, en los niveles limo arcillosos.

\begin{tabular}{ccccccc}
\hline Muestra & $\begin{array}{c}\text { Profundidad } \\
(\mathrm{m})\end{array}$ & Montmorillonita & Yeso & Anortita & Cuarzo & Calcita \\
\hline 4 & 0.3 & $\mathrm{X}$ & & $\mathrm{X}$ & $\mathrm{X}$ & $\mathrm{X}$ \\
6 & 0.5 & $\mathrm{X}$ & $\mathrm{X}$ & $\mathrm{X}$ & $\mathrm{X}$ & \\
10 & 0.75 & $\mathrm{X}$ & & $\mathrm{X}$ & $\mathrm{X}$ & $\mathrm{X}$ \\
21 & 1.51 & $\mathrm{X}$ & & $\mathrm{X}$ & $\mathrm{X}$ & $\mathrm{X}$ \\
42 & 2.9 & $\mathrm{X}$ & $\mathrm{X}$ & $\mathrm{X}$ & $\mathrm{X}$ & \\
75 & 6.3 & $\mathrm{X}$ & & $\mathrm{X}$ & $\mathrm{X}$ & $\mathrm{X}$ \\
97 & 8.7 & $\mathrm{X}$ & $\mathrm{X}$ & $\mathrm{X}$ & & $\mathrm{X}$ \\
100 & 8.9 & $\mathrm{X}$ & $\mathrm{X}$ & $\mathrm{X}$ & $\mathrm{X}$ & $\mathrm{X}$ \\
151 & 14.1 & $\mathrm{X}$ & & $\mathrm{X}$ & $\mathrm{X}$ & \\
175 & 16.55 & $\mathrm{X}$ & & $\mathrm{X}$ & & $\mathrm{X}$ \\
180 & 17 & $\mathrm{X}$ & & $\mathrm{X}$ & $\mathrm{X}$ & \\
212 & 22.2 & $\mathrm{X}$ & $\mathrm{X}$ & $\mathrm{X}$ & $\mathrm{X}$ & $\mathrm{X}$ \\
220 & 22.85 & $\mathrm{X}$ & $\mathrm{X}$ & $\mathrm{X}$ & $\mathrm{X}$ & \\
243 & 25.15 & $\mathrm{X}$ & & $\mathrm{X}$ & $\mathrm{X}$ & \\
259 & 27.1 & $\mathrm{X}$ & $\mathrm{X}$ & $\mathrm{X}$ & & \\
262 & 27.4 & $\mathrm{X}$ & $\mathrm{X}$ & $\mathrm{X}$ & $\mathrm{X}$ & \\
\hline
\end{tabular}

DRX $=$ Difracción de Rayos $X$

dominaban Poaceae, Cirsium, Brassicaceae $=$ Cruciferae y Apiaceae, elementos herbáceos que se desarrollan en ambientes de humedad (Xelhuantzi-López, 1994; SosaNájera, 2001).

Dentro del lago el conjunto de plantas acuáticas y subacuáticas (Figura 6) indica la existencia de un cuerpo de agua poco profundo caracterizado por Cyperaceae, Arenaria, Myriophyllum, Potamogeton, Lemna y Polygonum (Van Geel y Van Der Hammen, 1973; Lozano-García, 1989; Lozano-García y Ortega-Guerrero, 1993; Xelhuantzi-López, 1994; Andreex, 1998; Sosa-Nájera, 2001). Al inicio de este periodo existían aguas dulces de acuerdo con la presencia de algas como Coelastrum, que además sugieren aguas cálidas.

El registro de diatomeas como: Staurosira cf. construens, Nitzschia amphibia, Rophalodia gibba, y Epithemia spp., así como la presencia de COT, sugieren un lago no muy profundo con amplio desarrollo de vegetación acuática (Figura 4; Israde-Alcántara et al., 2002). En el sector central de esta zona la dominancia de Staurosira construens indica una tendencia a aguas diluidas en el lago y un posible aumento de la profundidad, que se hace mas evidente hacia la cima con la aparición de Stephanodiscus spp. y Aulacoseira spp.

En los resultados de los análisis mineralógicos se observa un nivel de yeso en la base de la zona 1, por lo que se infiere el dominio de un clima seco en la región (Figura 3; Engstron y Wright, 1984; Lozano-García y Ortega-Guerrero, 1993; Van Geel y Van der Hammen, 1973; Menking, 1997). En resumen, desde el comienzo de este intervalo se infiere que las condiciones climáticas fueron aumentando en humedad, hasta alcanzar condiciones más húmedas que las actuales.

\subsubsection{Zona II (26 m a $24.65 \mathrm{~m}$; ca. $98 \mathrm{ka}$ a ca. $93.5 \mathrm{ka}$ a.P.)}

Abarca un corto periodo de $c a .98 \mathrm{ka}$ a $c a .93 .5 \mathrm{ka}$ a.P. 

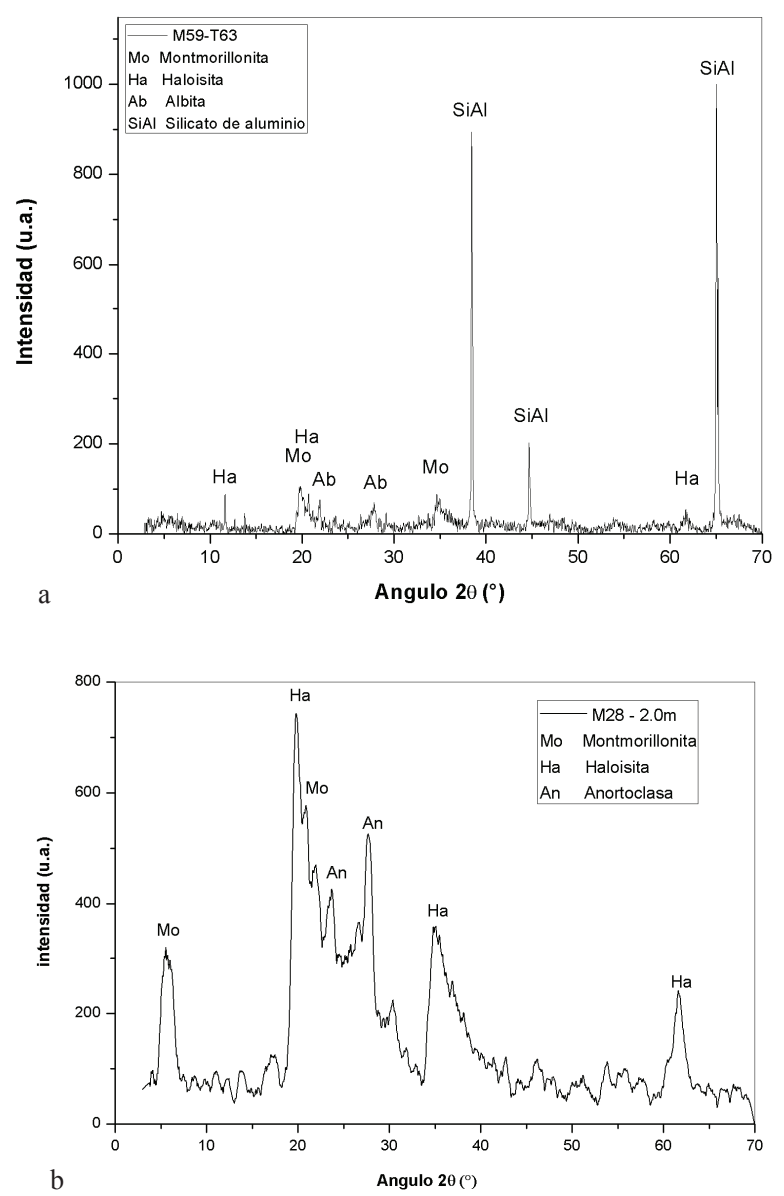

Figura 3. Análisis de DRX de la cima del núcleo a 27 y $2 \mathrm{~m}$ de profundidad.

lo que corresponde al inicio del último glacial. El registro está influenciado por la actividad volcánica (Figura 4) y la concentración polínica disminuye; en los espectros polínicos hay una dominancia de componentes del bosque de pino, lo que sugiere amplias zonas cubiertas por este tipo de vegetación. Las condiciones húmedas y frías están caracterizadas por Juglans, Alnus, Carpinus-Corylus, Abies mientras que los helechos (Bertini, 1994; González Medrano, 1996; Bertini et al., 1998) confirman un clima templado-húmedo que permitió el desarrollo de un bosque mesófilo (Orvis, 1998) en sitios protegidos como cañadas y pequeños valles de la región (Curry y Baker, 2000).

En el lago, Arenaria, Myriophyllum, Nymphaeaceae y Azolla sugieren condiciones frías, con una ligera recuperación del nivel lacustre hacia el final de esta zona, de acuerdo al incremento de Pediastrum y Staurosira cf. construens, ya que ambos organismos viven en un mayor tirante de agua (Nielsen y Sorensen, 1992; Israde-Alcántara et al., 2002; Figuras 4 y 6 ).

Los contenidos bajos de COT y de concentración polínica indican un lago con poca productividad biológica, mientras que el cuarzo y la anortita, que son producto del arrastre hacia la cuenca indican erosión a partir de la actividad volcánica en la región; cabe mencionar que no fue posible discernir cuando se trataba de depósitos de caída in situ o de epiclásticos. La ausencia de minerales indicadores de condiciones secas como calcita y yeso (Bengstson y Enell, 1986) apoyan la interpretación biológica de condiciones más húmedas que en la actualidad.

\subsubsection{Zona III (24. $65 \mathrm{~m}$ a $12.45 \mathrm{~m}$; ca. $93 \mathrm{ka} \mathrm{a} \mathrm{ca.} 53 \mathrm{ka}$ a.P.)}

La edad estimada para esta zona es de $c a .93 \mathrm{ka}$ a 53 ka a.P. y corresponde a la última glaciación. Conforme la glaciación avanza, cambian las condiciones húmedas que prevalecían en la región y que permitieron el desarrollo de bosques templados en la mayor parte de la cuenca y el clima se vuelve cada vez más frío y seco, ocasionando la modificación del paisaje hacia matorral (Figura 5). El conjunto palinológico apunta a condiciones abiertas, con la proliferación de elementos de hábitats abiertos xerófilos caracterizados por Poaceae, Ambrosia y algunas Compositae.

Localmente aparece vegetación hidrófila enraizada integrada por Cyperaceae (Martin, 1963; Sosa-Najera, 2001) y otros taxa como Polygonum, Arenaria, Potamogeton, Myriophyllum y Lemma. Los valores de Pediastrum en esta zona, indican un mayor espejo de agua (Lozano-García y Ortega-Guerrero, 1993; Mommerstecy et al., 1995), abundancia de nutrientes y temperaturas alrededor de 20 ${ }^{\circ} \mathrm{C}$, como también lo sugieren las algas acompañantes Botryococcus, Coelastrum, Mougeotia y Zygnemataceae (Figura 6). En esta misma zona Epithemia spp., Navicula cryptocephala, Rhopalodia gibberula y $R$. gibba ceden transicionalmente el hábitat al dominio de Staurosira cf. construens que en conjunto con el ligero aumentode los valores de Pediastrum, $\mathrm{ca}$. 88 ka a.P., sugieren una pequeña fase en la que se incrementan los niveles lacustres (LozanoGarcía y Ortega-Guerrero, 1993; Israde-Alcántara et al., 2002). El conjunto de paleoindicadores se asocia a un clima generalmente seco con algunos periodos de disturbio volcánico.

\subsubsection{Zona IV (12.45 m a $6.85 \mathrm{~m}$; ca. $53 \mathrm{ka} \mathrm{a} 35 \mathrm{ka}$ a.P.)}

Abarca de ca. 53 ka a 35 ka a.P. (Figura 2). El centro de este intervalo ha sido fechado en $42.4 \mathrm{ka}$ a.P. e incluye parte de la última glaciación. El registro polínico es muy fluctuante, pero se puede observar que hacia la parte media ocurre un descenso en el espectro arbóreo, que se recupera paulatinamente hacia la cima de la zona. Condiciones de mayor humedad y un aumento en la temperatura se infieren en la base de esta zona, lo que permite el restablecimiento de: Alnus, Salix, Juglans y Fraxinus como integrantes de bosques húmedos. Mientras que para las áreas bajas de la cuenca, se establece el desarrollo de comunidades microtérmicas y xéricas, en un clima semiseco, en donde se desarrolla un matorral arbóreo con Bursera, Agavaceae, Ipomoea, taxa característicos de matorral acompañados con 


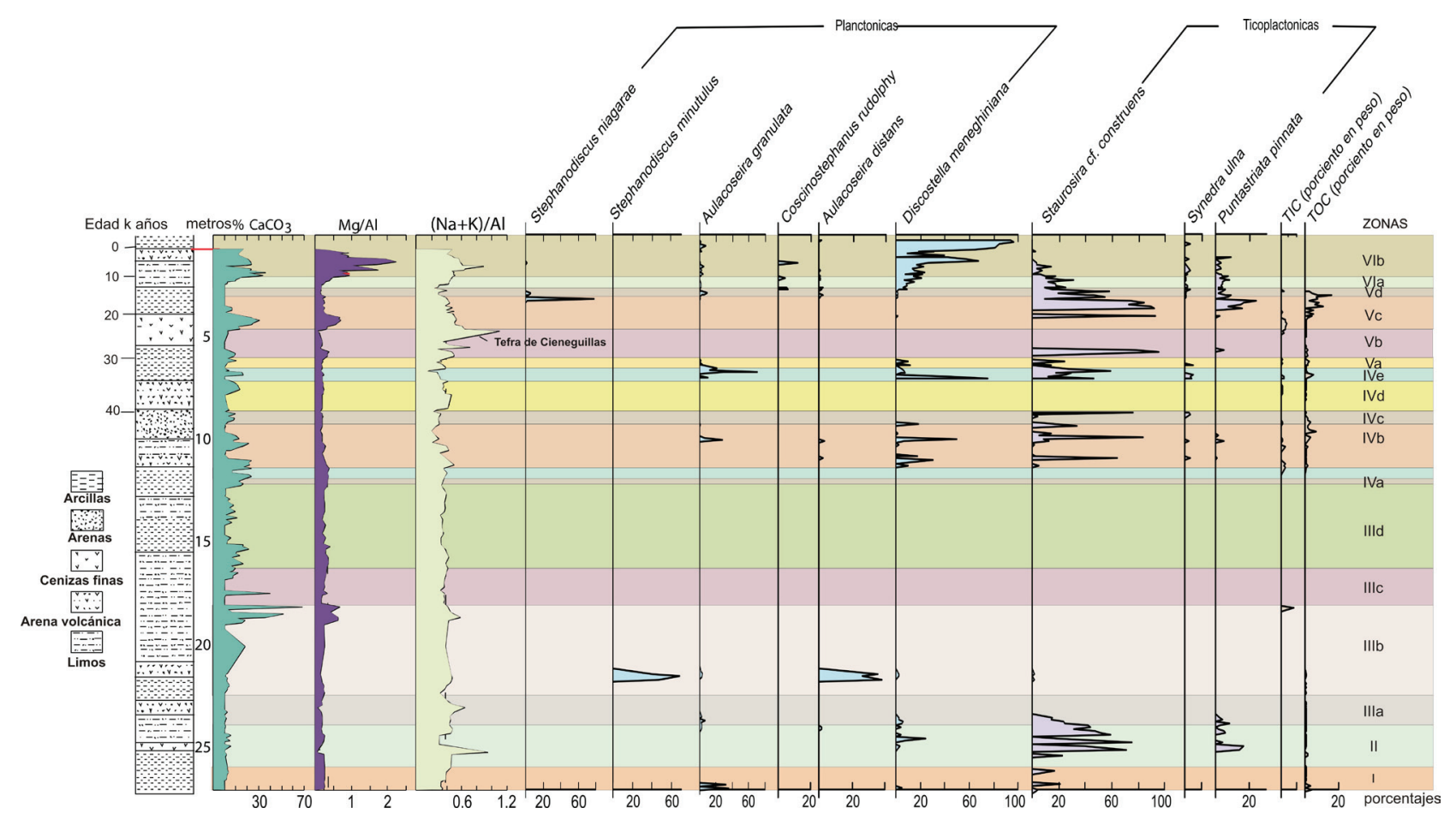

Figura 4. Relación $\mathrm{CaCO}_{3}, \mathrm{Mg} / \mathrm{Al},(\mathrm{Na}+\mathrm{K}) / \mathrm{Al}$ y TIC TOC y diatomeas en el núcleo de Cuitzeo.

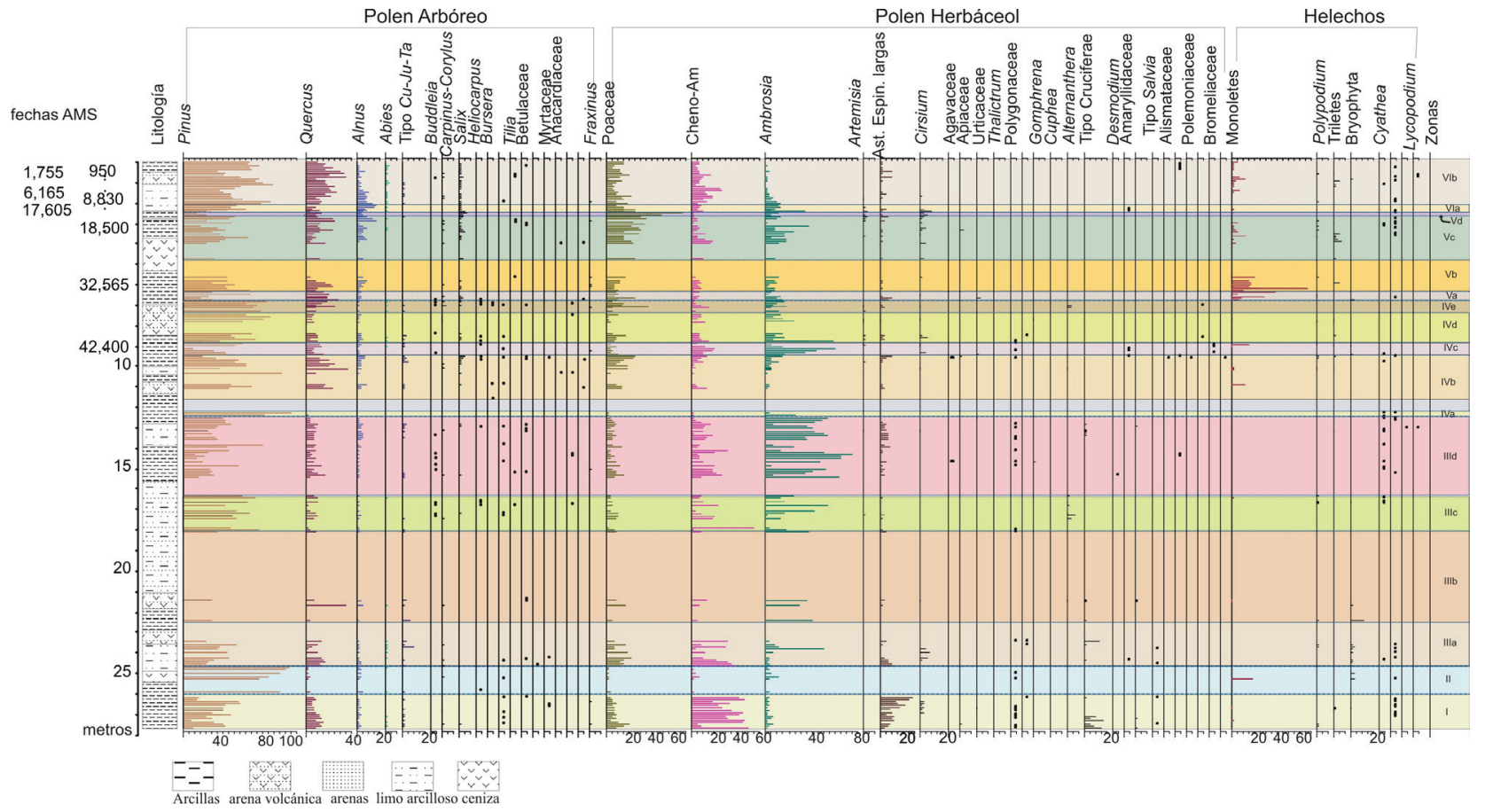

Figura 5. Registro polínico regional de la cuenca de Cuitzeo.

herbáceas como: Ambrosia, Cheno-Am y Poaceae (Martin, 1963; Rzedowski, 2005).

Localmente el lago, adquiere su tirante de agua más alto con respecto a periodos anteriores, indicado por valores muy altos de Pediastrum (ca. 10000 conteos), mientras en las riberas prosperaban los tulares (Figura 6). El registro de diatomeas es dominado por especies planctónicas y ticoplanctónicas: Aulacoseira granulata, Staurosira cf. 


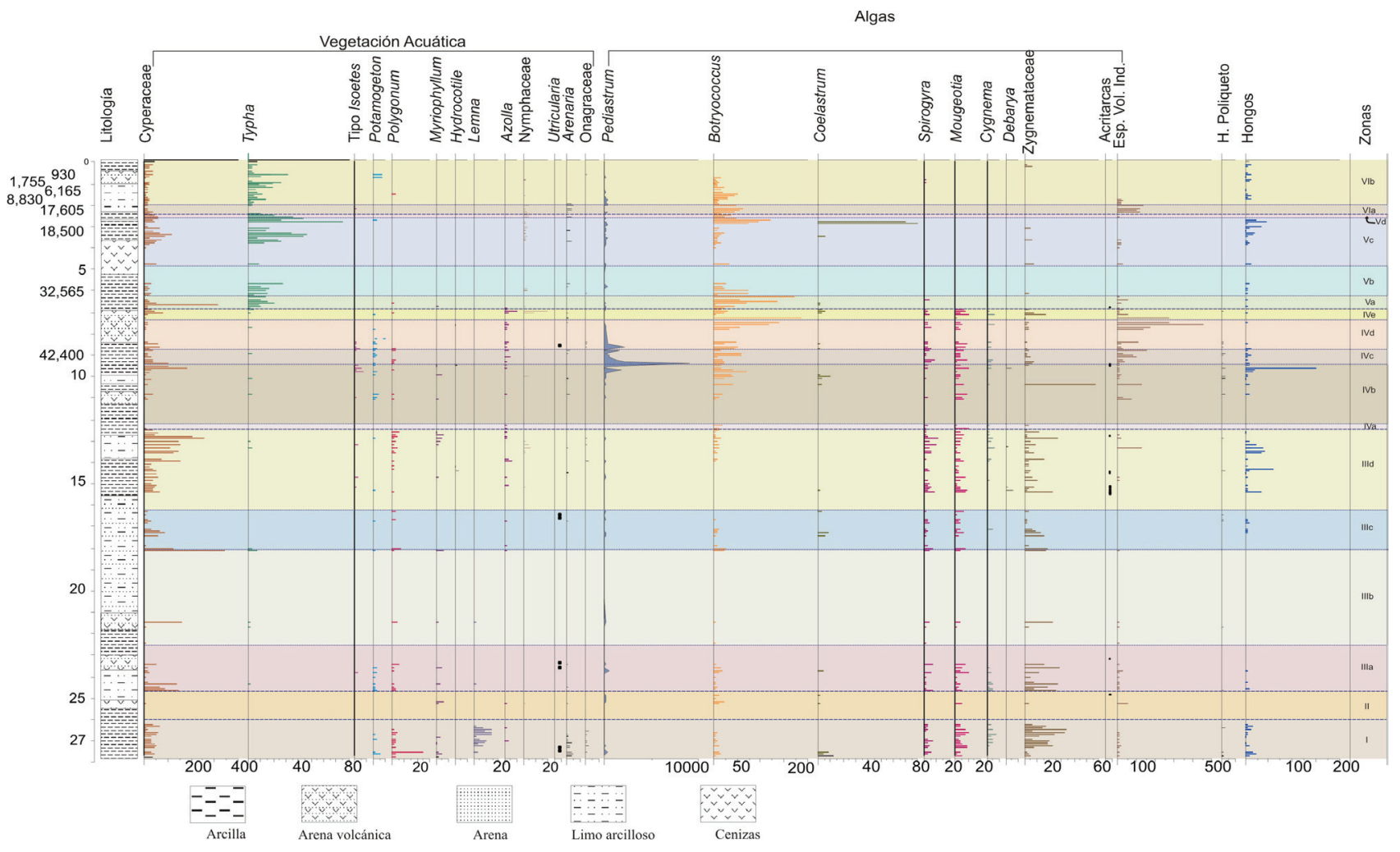

Figura 6. Registro polínico local del lago de Cuitzeo.

construens incluyendo la presencia de Stephanodiscus niagarae (Figura 4). Sin embargo, algunos niveles contienen especies perifiticas (Anomoeoneis sphaeropora y Cocconeis placentula) que evidencian lo fluctuante del lago para esa época.

\subsubsection{Zona V (6.8 m a $2.45 \mathrm{~m}$; ca $35 \mathrm{ka} \mathrm{a} 18 \mathrm{ka}$ a.P.)}

La cima de este intervalo comprende la última glaciación, incluyendo del glacial temprano al glacial tardío. En esta zona no aparece una representación completa de los conjuntos polínicos y de diatomeas, ya que se presenta una fase de actividad volcánica que interrumpe el registro. Sin embargo, la presencia de elementos herbáceos y arbustivos como Poaceae, Cheno-Am, Ambrosia, Asteraceae Espinas Largas, Cirsium y Thalictrum en especial hacia la cima de la zona que evidencian un deterioro ambiental ocasionado por la perturbación provocada por la actividad volcánica. El incremento del polen de vegetación de galería sugiere una mayor representación de esta comunidad vegetal hacia la parte superior de la zona (Figura 5).

Por su parte, el lago es muy fluctuante con registro alternado de elementos de pantano, vegetación halófila y algas indicadoras de aguas poco eutróficas (Martin, 1963; González Quintero y Fuentes Mata, 1980; Lozano-García y Ortega-Guerrero, 1993; Sosa-Najera, 2001). Dentro del contexto de la flora de diatomeas que habitaban el lago, se alcanzan los valores más altos de Anomoeoneis sphaeropora y se detectan apariciones de Campilodiscus clypeous, las cuales, sobre todo la segunda requieren condiciones de alta concentración iónica para su desarrollo.

Son evidentes dos pulsos de humedad, entre $c a .12 \mathrm{ka}$ y $20 \mathrm{ka}(2.0 \mathrm{~m}$ a $3.50 \mathrm{~m})$ y $c a .31 \mathrm{ka} \mathrm{a} 33 \mathrm{ka}(5.50 \mathrm{~m}$ a 6.80 $\mathrm{m}$ ) indicados por los altos valores de COT y un aumento de Staurosira construens y Stephanodiscus. Al final del Glacial tardío Staurosira construens decrece bruscamente al igual que el polen arboreo y no arboreo y es substituida hacia el Youngher Dryas por la breve pero muy notoria aparición de Stephanodiscus niagarae, a $2.80 \mathrm{~m}$ donde representa casi el $80 \%$ del total de las diatomeas. En este episodio se observan los valores más bajos de $\mathrm{CaCO}_{3}$ (Figura 4).

\subsubsection{Zona VI (2.45 m- a $0 \mathrm{~m}$; ca $17.5 \mathrm{ka}$ al presente.)}

Abarca una edad aproximada de $c a .17 .5 \mathrm{ka}$ al presente, incluyendo la fase Tardiglacial. Es en este tiempo cuando el lago y la cuenca adquieren las características actuales. Se observa polen de pino y claramente el incremento de encino, ambos más adaptados a suelos poco profundos y de humedad (Bello-Gonzalez y Labat, 1987). Respecto a los tipos polínicos de bosques de galería, Alnus experimenta un decremento progresivo muy marcado, mientras Salix mantiene sus valores muy similares a los de la zona anterior (Figura 5). La evidencia herbácea de Poaceae y Cheno- 
Am es muy fluctuante, mientras que Ambrosia marca una tendencia hacia la disminución durante el Holoceno, al parecer como consecuencia del cambio en el clima (Bertini, 1994; Cox y Moore, 1994; Bertini et al., 1998; Bradbury, 2000).

Entre $c a .15 \mathrm{ka}$ y $10 \mathrm{ka}$, los valores bajos de CIT y de $\mathrm{Mg} / \mathrm{Al}$ indican que el cuerpo de agua que continúa presentando dilución, con una tendencia a bajos niveles lacustres hasta el Holoceno medio, cuando el lago desciende en su nivel hasta adquirir las condiciones actuales, con áreas pantanosas ocupadas por: Typha y Cyperaceae (Martín, 1963; González Quintero y Fuentes Mata, 1980; Lozano-García y Ortega-Guerrero, 1993; Sosa-Nájera, 2001; Figura 6). Botryococcus sugiere aguas ricas en nutrientes, disminuyendo paulatinamente hasta desaparecer del registro hacia la actualidad. Discostella meneghiniana se incrementa hasta porcentajes de $95 \%$, subrayando el incremento de condiciones de turbidez y concentración iónica; mientras que las diatomeas perifíticas Nitzschia amphibia, Amphora lybica, Anomoeoneis sphaerophora y Navicula criptocephala sugieren un descenso del nivel del lago (Figura 4).

Las características muy similares durante el Holoceno medio son las asociadas en los valores de COT (Figura 4) y fungosporas, además de la sedimentación de halloysita, montmorillonita y anortoclasa (Figura 3b). La primera observada en fases de clima seco, mientras que las dos últimas provienen de fases de erosión en la cuenca (Engstron y Wright, 1984; Menking, 1997; Calvo et al., 2001). Un fuerte componente autigénico en este periodo es evidenciado por los altos valores de $\mathrm{Mg} / \mathrm{Al}$ observados.
3.2 Reconstrucción paleoambiental de las facies proximales

\subsubsection{Análisis de macropartículas carbonizadas}

Se realizaron cuatro trincheras en la zona norte del lago sobre la planicie de inundación actual, para determinar la influencia de los incendios en la cuenca en las zonas proximales. Este análisis muestra una fuerte correlación entre la manifestación de los incendios con la actividad volcánica, que la zona sufrió durante el Pleistoceno terminal. Esto se debe a que los incendios más grandes registrados para la zona norte están asociados a depósitos de caída y surges, mientras los incendios son ocasionados por la sequía y los cambios climáticos que fueron de menor intensidad, como se evidencia por el número de partículas carbonizadas asociadas a este tipo de eventos. De manera general, se observan tres incendios masivos (más de 300 partículas carbonizadas) uno coincidente con el vulcanismo de Los Azufres, otro ocurrido a los ca. $12 \mathrm{ka}$ a.P. y otro, muy intenso, ocurrido a los ca. 4 ka a.P. Conforme se avanza hacia el Holoceno terminal la actividad volcánica cesa y con ella la ocurrencia de incendios. No se observa una relación directa entre la actividad antropogénica y la ocurrencia de incendios (Figura 7).

\subsubsection{Análisis de diatomeas y facies sedimentarias}

Las facies en este sector son más detríticas de texturas areno-limosas y limo-arcillosas, con escasa madurez textural en las facies arenosas. Estas últimas son comunes en la trinchera del sector más al norte del lago. En la base de las secuencias fluviolacustres se intercalan materiales epiclásticos, surges depositados en agua y depósitos de

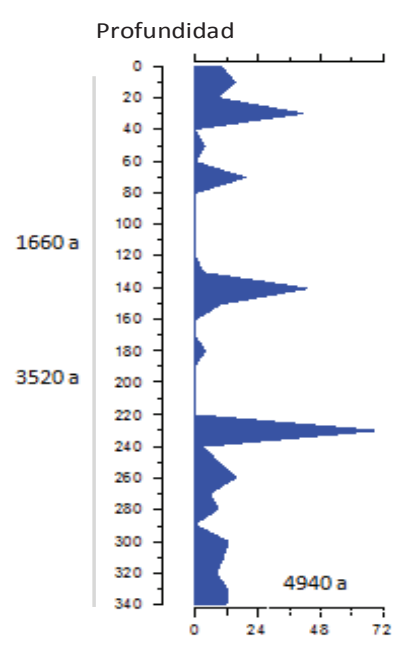

No. de partículas

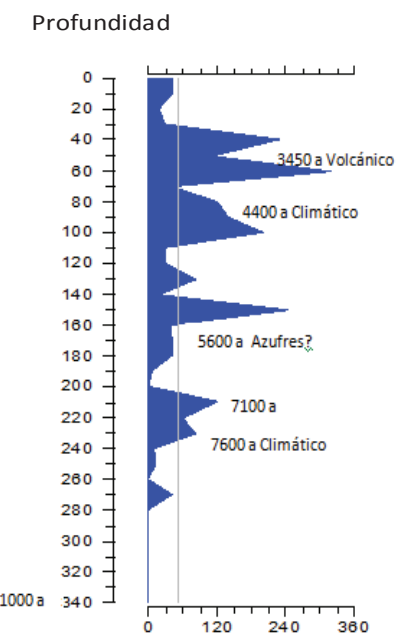

No. de partículas

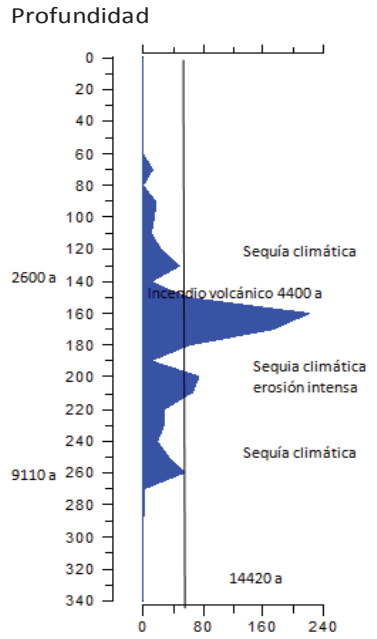

No. de partículas

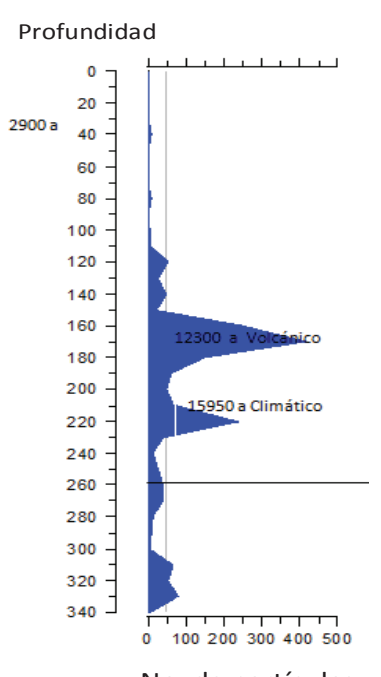

No. de partículas 


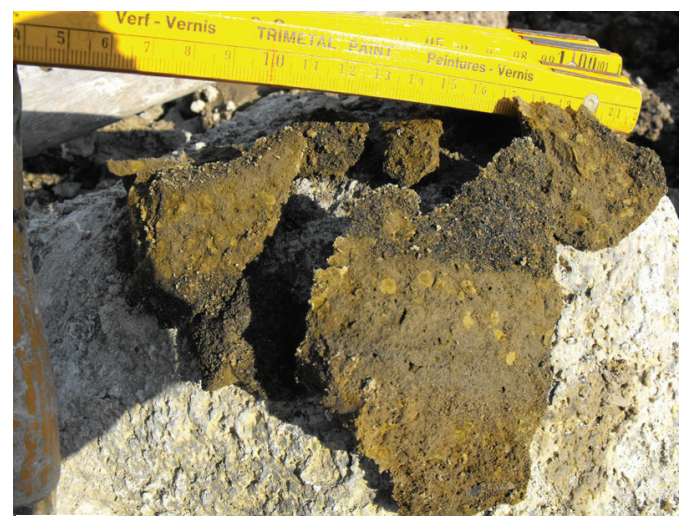

a

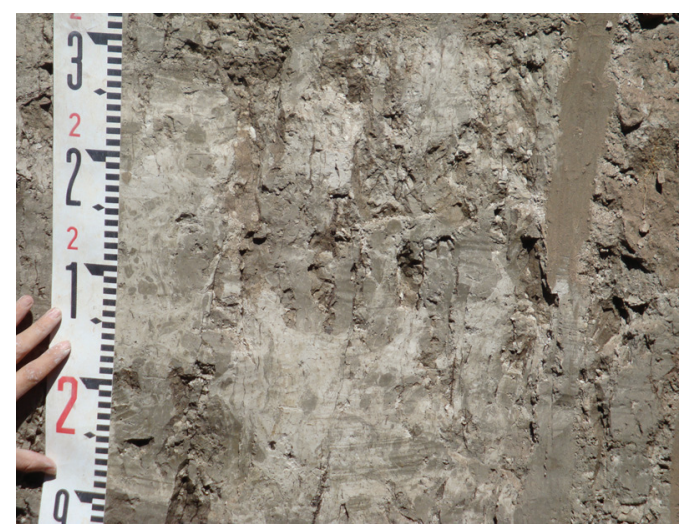

c

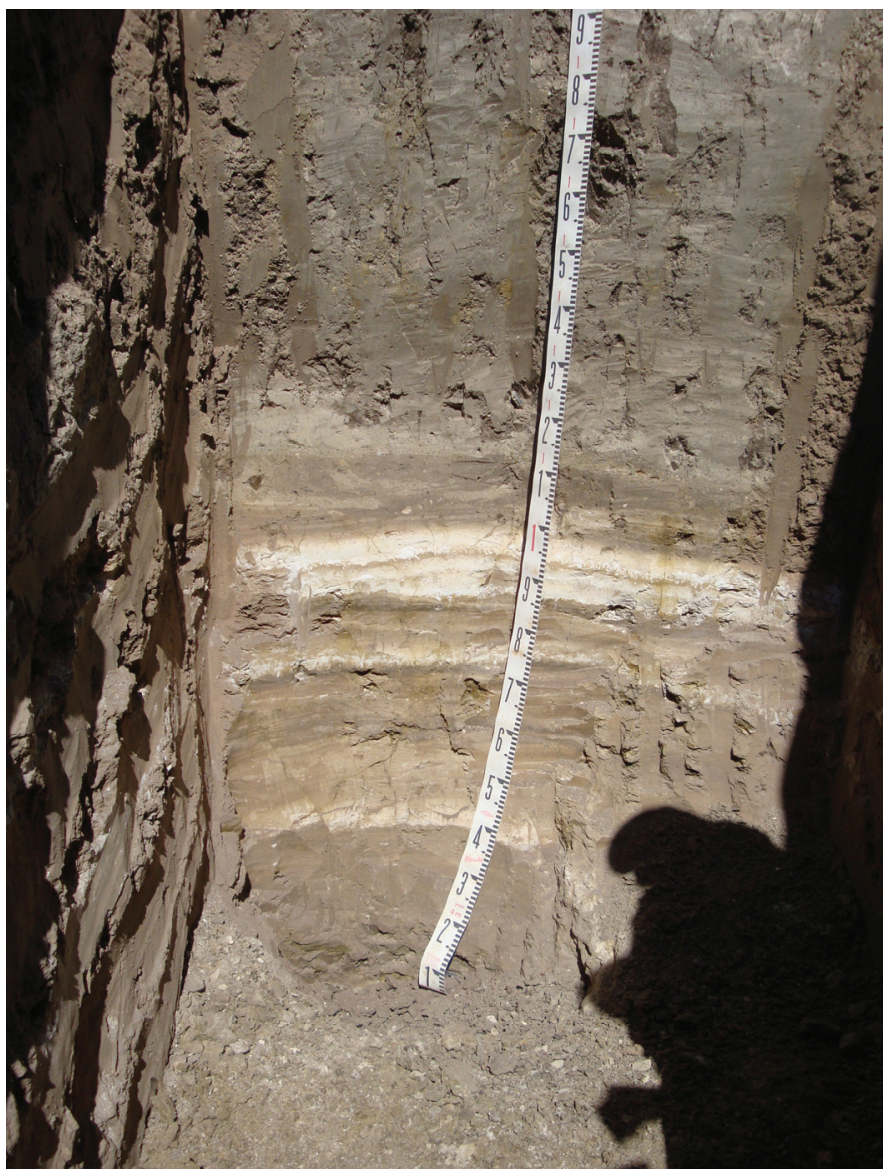

b

Figura 8. Trinchera tipo del norte del lago de Cuitzeo; 8a, Nivel de arena volcánica en contacto con paleosuelo pedogenizado; 8b, corte de la trinchera mostrando las facies volcánicas que pasan a fluviales y de borde lacustre inundado con intercalaciones de cenizas depositadas en agua y lentes de arena gruesa; 8c, facies limo-arcillosas deformadas.

caída. Sobre estos depósitos se observan limos arenosos color verdoso que presentan una pedogénesis que incluye pellets en paleosuelos alterados (Figura 8a). Se observan canales de relleno restringidos hacia las zonas más cercanas a los altos morfológicos que cambian de facies hacia una planicie aluvial en el borde antiguo del lago. Sobreyaciendo las facies arenosas se encuentran en discordancia erosional con limos, arenas y cenizas finas de color blanco (Figura $8 b)$ cubiertas por arcillas limosas correspondientes a depósitos de una planicie de inundación con cierto oleaje en coincidencia con el ingreso de pequeños canales fluviales. Se observan estructuras primarias cómo láminas, flammes $\mathrm{y}$ ondulitas formadas en un bajo régimen de corriente. Se observan algunos niveles oxidados en los depósitos arenosos redepositados que se reinyectan sucesivamente. Fenómenos sin-sedimentarios posiblemente asociados a sismicidad producen remoción de los limos arcillosos (Figura 8c).

Sobreyaciendo a los limos arcillosos se encuentran facies limo arcillosas de ciénega. Destacan los contactos netos entre las diferentes facies. En las facies limosas y limo-arenosas son comunes los restos de molares de équidos y pequeños mamíferos, indicando una ciénega inundada episódicamente. El registro de polen y diatomeas en las trincheras es escaso sugiriendo una intensa erosión que no permitió su preservación, o bien que no mantuvo el lago en esta región una estabilidad lacustre para permitir el desarrollo de flora. La primera hipótesis es la más congruente ya que las escasas diatomeas presentan alta fragmentación y no se observan fenómenos de disolución en los poroides además de la ausencia de polen. Sólo en una la trinchera de Buenavista, la flora de diatomeas está dominada por: Cocconeis placentula, Gomphonema parvulum, Cymbella spp., Cyclotella meneghiniana, Anomoeoneis sphaeropora, Epithemia túrgida, Amphora ovalis, Campilodiscus clypeus y Surirella spp., con Eunotia spp. como especie acompañante, sugiriendo el establecimiento de charcas someras en las zonas proximales del lago. Estas charcas poco profundas se caracterizan por tener abundante vegetación acuática y presentar un $\mathrm{pH}$ de intermedio a bajo. Campilodiscus clypeous y Surirella spp indican tendencias 
de alta concentración iónica en las charcas de esta planicie de inundación. Las variaciones en la preservación de los frústulos indica fenómenos de enriquecimiento en sales y aumento muy brusco de la alcalinidad, que produce la disolución de las valvas y está relacionado a procesos tafonómicos y a cambios en la concentración de $\mathrm{Mg}, \mathrm{Ca}$ y $\mathrm{K}$, como se distingue hacia el núcleo del centro del lago, además de una remoción constante de los sedimentos.

En las trincheras, no obstante que las diatomeas son muy escasas, son muy afines litológicamente con las del centro del lago de Cuitzeo, indicando una conexión estacional con la zona norte. Los frústulos presentan evidencias de corrosión y retrabajamiento intenso, incluso en Cymbella que es un género con una valva robusta que normalmente se preserva en condiciones de alta energía fluvial y aluvial.

\subsubsection{Paleoambientes inferidos en la zona litoral}

Se observa que este hábitat más tendiente a aluvial era bañado estacionalmente por charcas muy someras y efímeras que no permitían el desarrollo de comunidades de diatomeas. Se trata de una planicie aluvial que pasaba a un lago somero episódicamente y al que llegaron flujos piroclásticos con alta temperatura que incineraron la cubierta vegetal.

En el primer episodio de transgresión en la zona, la concentración iónica elevada del hábitat lacustre cercano permitió el depósito de flora que tiene preferencia por aguas salinas, sugiriendo que el cuerpo de agua cercano era muy somero. Hacia el Holoceno medio, espículas de esponja y fitolitos documentan un ambiente tendiente a seco con abundantes pastos en el entorno. Estas charcas y planicies de inundación fueron cubiertas por cenizas volcánicas y materiales epiclásticos provenientes de zonas cercanas.

\subsection{Correlación entre los Lagos de Cuitzeo, Pátzcuaro y Zacapu}

Los estudios más recientes que han abordado la reconstrucción paleoambiental en la región (Corredor Tarasco) corresponden a los lagos de Cuitzeo (VelázquezDurán et al., 2001; Israde-Alcántara et al., 2002), Pátzcuaro (Watts y Bradbury, 1982; Bradbury, 2000) y Zacapu (Metcalfe y O’Hara, 1992, Xelhuantzi-López, 1994, 1995; Ortega et al., 2002). El análisis de diatomeas en la secuencia de Cuitzeo (Israde-Alcántara et al., 2002) sugiere que el lago de Cuitzeo ha mantenido aguas dulces, alcalinas y de cierta profundidad, con Staurosira spp. como dominante. Por otro lado, se documenta la existencia de dos pequeñas fases húmedas durante el intervalo correspondiente a $c a$. 42 ka a 40 ka a.P. y la segunda $c a .20$ ka a 15 ka a.P., con Stephanodiscus spp. y Aulacoseira spp., como dominantes. Entre $35 \mathrm{ka}$ y $18 \mathrm{ka}$ las evidencias de los conjuntos polínicos $\mathrm{y}$ de diatomeas sugieren condiciones con tendencia a secas semejantes a las observadas en las cuencas de Zacapu, Chalco y Lerma, al occidente y al oriente del lago de Cuitzeo (Caballero y Ortega, 1998).
En el ex-lago de Zacapu, Ortega et al. (2002) detectan condiciones relativamente húmedas entre 52 ka y 35 ka y tendencia a condiciones secas hacia los 25 ka que culminan con un hiatus en la sedimentación. En el lago de Cuitzeo una pequeña fase húmeda se observa también $c a .50 \mathrm{ka}$ y durante el último máximo glacial entre $c a .23$ ka y 18 ka. La actividad volcánica de la región es muy constante interrumpiendo el registro de la sedimentación. Sin embargo, por la presencia de marcadores preservados en algunos niveles hay evidencia de que estos ambientes secos continúan durante el máximo glacial. Posteriormente en el Glacial tardío, entre $c a .18 \mathrm{ka} \mathrm{y} 12 \mathrm{ka}$, altos porcentajes de COT y la presencia de $S$. niagarae indican un mejoramiento climático, que viene seguido por una fase seca y después de nuevo por una tendencia a húmeda en el inicio del Holoceno medio donde valores más altos de COT indican tiempos de residencia del agua más largos que los observados previamente.

En la exciénega de Zacapu, el análisis palinológico del Holoceno reporta ambientes templados (Xelhuantzi-López; 1994; 1995). Se observa un clima templado húmedo o subhúmedo entre $8.200 \mathrm{ka}$ y $7.4 \mathrm{ka}$, mientras en Cuitzeo se reconoce un clima templado subhúmedo en la parte alta y templado, seco en las áreas bajas y colindantes con el lago. Entre $c a .7 .4$ y $4.7 \mathrm{ka}$ en Zacapu se reporta un clima semi-seco templado, mientras que para Cuitzeo el patrón climático presenta una tendencia hacia un clima templado subhúmedo. Por último, de 4.7 ka al presente, XelhuantziLópez (1995) menciona un clima subhúmedo y templado para Zacapu, mientras que para Cuitzeo la curva climática parte de un clima templado semiseco hacia un clima templado subhúmedo que declina en las últimas décadas hacia un ambiente más seco.

En el lago de Pátzcuaro, Bradbury (2000) sugiere un clima templado seco de $48 \mathrm{ka}$ a $10 \mathrm{ka}$ a.P. y templado subhúmedo para los últimos 10 ka a.P. Sin embargo, RoblesCamacho et al. (2009) registran a 26 ka a.P. condiciones húmedas y frías que permitieron el desarrollo de un bosque mesófilo asociado a Liquidambar, Juglans, Betula y Corylus.

Del Holoceno medio al presente el lago de Cuitzeo, ha experimentado procesos de erosión y descenso del nivel lacustre, evidenciados por la ausencia de registro polínico y de diatomeas en la zona proximal del lago en su porción norte. No obstante estas tendencias a climas secos también se detectan pulsos de humedad con incrementos en el espejo de agua.

\section{Conclusiones}

A pesar de que los análisis se integran a partir de un solo núcleo y cuatro trincheras en un lago tan extenso, se ofrece un panorama del impacto del cambio climático durante el Pleistoceno Tardío y el Holoceno. Cabe señalar que el vulcanismo y la erosión sucesiva han provocado 
complejas variaciones en la red hidrológica, en los patrones de sedimentación hacia el lago y han inhibido el depósito de polen y diatomeas; principalmente desde $c a .80 \mathrm{ka}$ a 33 ka a.P. y de $c a .20$ ka a 24 ka. Sin embargo, otros indicadores, tales como los altos valores de $\mathrm{CaCO}_{3}$ y de CIT, sugieren un lago cerrado y un descenso del nivel lacustre. Estas características también se observan hacia las facies más proximales del lago en sus bordes norte y sur, donde además se presentan sedimentos finos pertenecientes a planicies aluviales con rellenos de canal que son invadidos episódicamente por eventos transgresivos durante el Holoceno medio. La actividad volcánica se detecta en las bases de la mayoría de las trincheras, incluyendo surges de alta temperatura y cenizas volcánicas depositados en agua.

En la zona norte del lago de Cuitzeo, sobre estos materiales existe una intensa erosión que mezcla los sedimentos evitando su interpretación. A lo largo de los últimos $c a .100 \mathrm{ka}$ a.P se han detectado cinco fases de humedad en la cuenca de Cuitzeo, la primera hace $c a .100$ ka, la segunda $c a$. $50 \mathrm{ka}$, la tercera de $c a .44 \mathrm{ka} \mathrm{a} 42 \mathrm{ka}$, la cuarta entre $c a .31 \mathrm{ka}$ y $32 \mathrm{ka}$ y la última al final del último glacial máximo y durante el Glacial tardío, entre ca. 20 y $14 \mathrm{ka}$, donde se presentan altos valores de COT y los más bajos de $\mathrm{Ca}$, indicando un lago de aguas diluidas con tendencia a ser profundas. Durante el Holoceno medio los altos valores de $\mathrm{COT}$ y de $\mathrm{CaCO}_{3}$ indican tiempos de residencia del agua más largos. El Holoceno tardío está representado por diatomeas de aguas más concentradas en sales. La desaparición de Botryococcus e incremento de Discostella meneghiniana, evidencian una somerización del lago con un gran aporte de material sólido, aguas turbias y eutróficas que marcan el establecimiento de las condiciones ambientales actuales en el lago.

En las zonas proximales del lago se observan abundantes restos de partículas carbonizadas, cuyas proporciones más altas están ligadas a procesos de surges a altas temperaturas y a algunos eventos climáticos. El Holoceno medio y tardío presentan escasos registros de incendios, lo que estaría indicando poca importancia de la actividad agrícola durante este periodo, lo cual podría significar que los pueblos asentados en Cuitzeo dependían más de las actividades propias del lago, que de las actividades agrícolas. Esto lleva a reconsiderar las relaciones que los antiguos habitantes mantenían con los ambientes acuáticos, la cual aparentemente pudo ser más estrecha de lo que se ha considerado hasta ahora.

\section{Agradecimientos}

Nuestro profundo agradecimiento a Luis Rubio Lepe, quien estuvo presente durante la perforación del núcleo a lo largo de Cuitzeo e inició los estudios de geoquímica. Esta investigación está dedicada a su memoria.

\section{Referencias}

Andreex, A., 1998, Holocene vegetation history from a Pearland, Fairbanks AK, en $15^{\mathrm{TH}}$ Biennial Meeting, American Quaternary Associaton: Puerto Vallarta, México, AMQUA, Abstracts, 83.

Arredondo, F.J., 1983, Levantamiento gravimétrico en la zona central de la Laguna de Cuitzeo, Michoacán: México, D.F., Comisión Federal de Electricidad, Informe 29/83 G.P.G, 11 p.

Bello-Gonzalez, M.A., Labat, I.N., 1987, Los Encinos (Quercus) del Estado de Michoacán, México: Cuadernos de Estudios Michoacanos I, $212 \mathrm{p}$.

Bengston, L., Enell, M., 1986, Chemical analysis, en Benglund, B.E. (ed.), Handbook of Holocene Palaeoecology and Palaeohydrology: New York, John Willey and Sons, $869 \mathrm{p}$.

Bertini, A., 1994, Palynological investigations on Upper Neogene and Lower Pleistocene sections in central and northern Italy: Memorie della Società Geologica Italiana, 48, 431-443.

Bertini, A., Londeix, L., Maniscalco, R., Di Stefano, A., Suc, J.P., Clauzon, G., Gautier, F., Grasso, M., 1998, Paleobiological evidence of depositional conditions in the Salt Member, GessosoSolfifera Formation (Messinian, Upper Miocene) of Sicily: Micropaleontology, 44, 413-433.

Bradbury, J.P., 2000, Limnologic history of Lago de Pátzcuaro, Michoacán, México, for the past 48000 years: impacts of climate and man: Palaeogeography, Palaeoclimatology, Palaeoecology, 163, 69-95.

Caballero, M., Ortega Guerrero, B., 1998, Lake levels since about 40000 years ago at Lake Chalco, near Mexico City: Quaternary Research 50, 69-79.

Calvo, E., Villanueva, J., Grimalt, J., Boelart, A., Labeyrie, L., 2001, New insights into the glacial latitudinal temperature gradients in North Atlantic. Results from $\mathrm{U}^{\mathrm{k}}{ }_{37}$ sea surface temperatures and terrigenous inputs: Earth and Planetary Science Letters, 188, 509-519.

C.F.E (Comisión Federal de Electricidad), 1986, Muestra CU-8 Dataciones radiométricas en rocas del NW de Michoacán: México, D. F., Comisión Federal de Electricidad, Reporte 0010/86, 20 p.

Cox, B., Moore, D., 1994, Biogeography an ecological and evolutionary approach: Oxford, Inglaterra, Blackwell Scientific Publications, $326 \mathrm{p}$.

Curry, B.B., Baker G.R., 2000, Palaeohydrology, vegetation and climate since the late Illinois Episode $(\sim 130 \mathrm{ka})$ in south-central Illinois: Palaeogeography, Palaeoclimatology, Palaeoecology, 155, 59-81.

Davies, S.J., Metcalfe, S.E., Bernal-Brooks, F., Chacón-Torres, A., Farmer, J.G., MacKenzie, A.B., Newton, A.J., 2005, Lake sediments record sensitivity of two hydrologically closed upland lakes in Mexico to human impact: Ambio, 34, 470-475.

Engstron, R.D., Wright, H.E., 1984, Chemical stratigraphy of lake sediments as a record of environmental change, en Haworth, E.Y., Lund, J.W.G. (ed.), Lake sediments and environmental history: Minneapolis, Minnesota, EUA, University of Minnesota Press, 11-67.

Erdtman G., 1969, Handbook of palynology morphology-taxonomyecology, an introduction to the study of pollen grains and spores: New York, EUA, Hafner Publishing, 486 p.

Garduño-Monroy, V.H, Negrín, J.L. 1992, Magmatismo, hiatus y tectonismo de la Sierra Madre Occidental y del Cinturón Volcánico Mexicano: Geofísica Internacional, 31, 417-429.

Gasse, F., 1986, East African Diatoms. Taxonomy, ecological distribution: Bibliotheca Diatomologica Band 11: Berlin, Alemania, J. Cramer, $201 \mathrm{p}$.

González Medrano, F., 1996, Algunos aspectos de la evolución de la vegetación de México: Boletín de la Sociedad Botánica de México, $58,129-136$.

Gonzalez Quintero L., Fuentes Mata, M., 1980, El Holoceno en la porción central de la cuenca del Valle de México, en Coloquio Sobre Paleobotánica y Palinología: México, Instituto Nacional de Antropología e Historia (INAH), 86, 113-132.

Hartley, B., Barber, H.G., Carter, J.R., 1996, An Atlas of British diatoms: Bristol, Biopress Limited, $601 \mathrm{p}$. 
Hustedt, F., 1930, Bacillariophyta (Diatomae), in Pascher (ed.) Die Susswasserflora Mitteleuropa: Jena, Alemania, Gustav Fischer Verlag, $466 \mathrm{p}$.

Israde-Alcántara I., 1995, Bacini Lacustri dal Settore Centrale dall'arco vulcanico messicano. Stratigrafia ed evoluzione vulcanotettonica basata sulle diatomme: Milán, Università degli Studi di Milano, Tesis doctoral, $254 \mathrm{p}$.

Israde-Alcántara, I., Garduño-Monroy, V.H., 1999, Lacustrine record in a volcanic intra-arc setting: The evolution of the Late Neogene Cuitzeo Basin System (Central Western Mexico, Michoacán): Paleogeography, Palaeoclimatology, Palaeoecology, 151, 209-227.

Israde-Alcántara, I., Garduño-Monroy, V.H., Ortega-Murillo, R., 2002, Paleoambiente lacustre del Cuaternario tardío en el centro del lago de Cuitzeo: Hidrobiologica, 2, 61-78.

Israde-Alcántara, I., Ortega-Guerrero B., Caballero, M., Lozano S., Sánchez G., 2009, The last ca. 1000 years of evolution of laminated Tacámbaro crateric lake based on diatom and TOC and TIC record (resumen), en $11^{\text {th }}$ International Paleolimnology Symposium: Guadalajara, México, International Paleolimnology Association, 77.

Israde-Alcántara, I., Miller, W.E., Garduño-Monroy, V.H., Barron, J., Rodriguez-Pascua, M.A., 2010, Palaeoenvironmental significance of diatom and Vertebrate fossils from Late Cenozoic Tectonic Basins in west-central México: A review: Quaternary International, 219, 79-94.

Krammer, K., Lange-Bertalot, H., 1986, Bacillariophyceae 1. Naviculaceae. Süßwasserflora von Mitteleuropa: Stuttgart, Alemania, Gustav Fischer Verlag, $876 \mathrm{p}$.

Krammer, K., Lange-Bertalot, H. 1988, Bacillariophyceae 2. Bacillariaceae, Epithemiaceae, Surirellaceae. Süßwasserflora von Mitteleuropa : Stuttgart, Alemania, Gustav Fisher Verlag, 596 p.

Krammer, K., Lange-Bertalot, H., 1991a, Bacillariophyceae 3. Centrales, Fragilariaceae, Eunotiaceae. Süßwasserflora von Mitteleuropa: Stuttgart, Alemania, Gustav Fischer Verlag, 576 p.

Krammer, K., Lange-Bertalot, H., 1991b, Bacillariophyceae 4. Achnanthaceae. Kritische Ergänzungen zu Navicula (Lineolatae) und Gomphonema. Süßwasserflora von Mitteleuropa: Stuttgart, Alemania, Gustav Fischer Verlag, 437 p.

Laird, L.D., Campbell, I.D., 2000, High resolution palaeofire signals from Christina Lake, Alberta: a comparison of charcoal signals extracted by two different methods: Palaeogeography, Palaeoclimatology, Palaeoecology, 164, 111-123.

Lange-Bertalot, H., 2001, Navicula sensu stricto, 10 genera separated from Navicula sensu lato, Frustulia. Diatoms of Europe Vol. 2: Ruggell, Liechtenstein, Gantner Verlag, 526 p.

Lozano-García, S., 1989, Palinología y paleoambientes pleistocénicos de la cuenca de México: Geofísica Internacional, 28, 335-362.

Lozano-García, M.S., Ortega-Guerrero, B., 1993, Palynological and magnetic susceptibility records of Lake Chalco, central Mexico: Palaeogeography, Palaeoclimatology, Palaeogeography, 109, 177-191.

Martin, P.S., 1963, The Last 10,000 Years: A fossil pollen record of the American Southwest: Tucson, Arizona, EUA, The University of Arizona Press, $87 \mathrm{p}$.

Menking, K.M., 1997, Climatic signals in clay mineralogy and grainsize variations in Owens Lake core OL-92, Southeast California: Geological Society of America Special Paper, 317, 25-36.

Metcalfe, S.E., O'Hara, S.L., 1992, Sensibilidad de lagos mexicanos a alteraciones en el medio ambiente: ejemplos del eje Neovolcánico: Ingeniería Hidráulica en México, mayo-diciembre, 107-127.

Metcalfe, S.E., Davies, S.J., Braisby, J.D., Leng, M.J., Newton, A.J., Terrett, N.L., O'Hara, S.L., 2007, Long and short-term change in the Pátzcuaro Basin, central Mexico: Palaeogeography, Palaeoclimatology, Palaeoecology, 247, 272-295.
Mommerstecy, H.J.P.M., Loutre, M.F., Young, R., Wijmstra, T. A., Hooghiemstra, H., 1995, Orbital forced frequencies in the 975000 years pollen record from Tenagi Pilippon (Greece): Climate Dinamics, 11, 4-24.

Nielsen, H., Sorensen, I., 1992, Taxonomy and stratigraphy of late glacial Pediastrum taxa from Lysmosen, Denmark a preliminary study: Review of Palaeobotany and Palynology, 74, 55-75.

Ortega, B., Caballero, M., Lozano, S., Israde, I., Vilaclara, G., 2002, 52000 years of environmental history in Zacapu basin, Michoacan, Mexico: the magnetic record: Earth and Planetary Science Letters, 202, 663-675.

Ortega, B., Vázquez, G., Caballero, M., Israde, I., Lozano-García, S., Schaaf, P., Torres, E., 2010, Late Pleistocene-Holocene record of environmental changes in Lake Zirahuen, Central Mexico: Journal of Paleolimnology, 44, 745-760.

Orvis, K.H., 1998, Modern surface pollen from three transects across the southern Sonora desert margin, Northwestern Mexico: Palynology, $22,197-211$

Robles-Camacho, J., Corona-Chávez, P., Morales-Gámez, M., Guzmán, A.F., Domínguez-Vázquez, G., Israde-Alcántara, I., OliverosMorales, A., 2009, A Gomphothere from Lake Pátzcuaro, Michoacán, México: Current Research in the Pleistocene, 26, 172-174.

Round, F.E., 1981, The ecology of algae: Cambridge, Inglaterra, Cambridge University Press, $664 \mathrm{p}$.

Rumrich, U., Lange-Bertalot, H., Rumrich, M., 2000, Diatoms of the Andes (from Venezuela to Patagonia/Tierra del Fuego), Iconographia Diatomologica Vol. 9: Koenigstein, Hesse, Alemania, Koeltz Scientific Books, 673 p.

Rzedowski, J., 2005, Vegetación de México: México, D.F., Conabio, 416 p.

Sosa-Nájera, M.S., 2001, Registro palinológico del Pleistoceno TardíoHoloceno en el extremo meridional de la cuenca de México: paleoambientes e inferencias paleoclimáticas: México, D. F., Universidad Nacional Autónoma de México, Tesis de maestría, $143 \mathrm{p}$.

Stuiver, M., Reimer, P., 1993, Extended ${ }^{14} \mathrm{C}$ data base and revised Calib 3.0 ${ }^{14} \mathrm{C}$ calibration program: Radiocarbon, 35, 215-230.

Van Geel, B., Van der Hammen, T., 1973, Upper Quaternary vegetation and climatic sequence of the Fuquene area (eastern Cordillera, Colombia): Palaeogeography, Palaeoclimatology, Palaeoecology, 14, 9-92.

Velázquez-Durán, R., Israde-Alcántara, I., Lozano-García, S., 2001, Significado ecológico de los palinomorfos depositados en el lago de Cuitzeo, Michoacán, durante los últimos 35000 años: Ciencia Nicolaita, 29, 45-61.

Watts, A.W., Bradbury, J.P., 1982, Paleoecological studies at Lake Patzcuaro on the west-central Mexican Plateau and at Chalco in the basin of Mexico: Quaternary Research, 17, 56-70.

Xelhuantzi-López, M.S., 1994, Estudio palinológico de cuatro sitios ubicados en la Cuenca de Zacapu: fondo de ciénega, contacto Lomas-Ciénega, pantano interno y Loma Alta, en Petrequin, P. (ed.) 8000 años de la cuenca de Zacapu, evolución de los paisajes y primeros desmontes: Morelia, Michoacán, Cuadernos de Estudios Michoacanos, 6, 81-93.

Xelhuantzi-López, M.S., 1995, Palynologie et palèoenvironnement du bassin de Zacapu, Michoacán, Mexique, depuis 8000 ans: Geofísica Internacional, 34, 239-248

Manuscrito recibido: Julio 10, 2009.

Manuscrito corregido recibido: Agosto 20, 2010.

Manuscrito aceptado: Octubre 10, 2010. 